\title{
Geometrical Tunability and Local Electric Field Distribution of Gold-dielectric-silver three-layered Cylindrical Nanotube
}

YeWan Ma ( $\sim$ mayewan@126.com )

Anqing Normal University https://orcid.org/0000-0003-1916-9124

\section{Zhao-Wang Wu}

Anqing Normal University

Juan Li

Anqing Normal University

Yan-Yan Jiang

Anqing Normal University

Xun-Chang Yin

Anqing Normal University

Ming-Fang Yi

Anqing Normal University

Li-Hua Zhang

Anqing Normal University

\section{Research Article}

Keywords: Localized surface plasmon resonance (LSPR), Gold-dielectric-silver nanotube, Quasi-static approximation, Plasmon hybridization theory

Posted Date: February 23rd, 2021

DOI: https://doi.org/10.21203/rs.3.rs-256616/v1

License: (a) (1) This work is licensed under a Creative Commons Attribution 4.0 International License.

Read Full License 


\title{
Geometrical Tunability and Local Electric Field Distribution of Gold-dielectric-silver three-layered Cylindrical Nanotube
}

\author{
Ye-Wan Ma*, Zhao-Wang Wu, Juan Li, Yan-Yan Jiang, Xun-Chang Yin, Ming-Fang Yi, and Li-Hua Zhang \\ School of Mathematics and Physics, Anqing Normal University, Anqing 246133, People's Republic of China
}

\begin{abstract}
In this paper, the effects of inner core radius, shell thickness, the dielectric function of middle nanoshell and surrounding medium on localized surface plasmon resonance (LSPR) of gold-dielectric-silver nanotube are studied based on the quasi-static approximation. Theoretical calculation results show that LSPR of gold-dielectric-silver nanotube and LSPR numbers can be well optimized by tuning the geometrical parameters. The longer wavelength of $\left|\omega_{-}^{-}\right\rangle$mode takes place a distinct red-shift with an increase in the inner core radius and the thickness of middle dielectric layer, while a blue-shift with increasing outer shell thickness. The physical mechanisms are investigated based on induced charges, plasmon hybridization theory and phase retardation. In addition, the effect of middle dielectric function and surrounding medium on LSPR, the local electric field distribution are also reported. Our study provides a way to analyze and broaden the applications of golddielectric-silver nanotube.

Key words: Localized surface plasmon resonance (LSPR), Gold-dielectric-silver nanotube, Quasi-static approximation, Plasmon hybridization theory
\end{abstract}

\section{INTRODUCTION}

Special optical properties of noble metal nano-structures (i.g., gold and silver) demonstrate different optical properties in comparison with bulk metal materials, thus optical properties of nobel metal nano-structures have attractive interest due to the intense light absorption of localized surface plasmon resonance (LSPR). LSPR originates from the collective oscillation of conduction electrons in accordance with the incident wave frequency[1,2], the results show that both the intensity and LSPR peak position can be well tuned by changing the nano-structure size, shape, structure and surrounding medium function[3, 4]. Therefore, the LSPR can be well tuned by adjusting the parameters of the particle size, shape, component and surrounding medium function[5]. LSPR has been widely used in chemical and biological sensing[6] and surface-enhanced Raman spectroscopy (SERS)[7]. The studies show that core-shell nano-particle is one of the most efficient methods to vary the LSPR wavelength from ultraviolet to infrared region, optical properties of nanoshells are explained by plasmon hybridization theory[8]. Xia et al have synthesized three-layered gold-silica-gold nanoshell with a size sub-100nm, the results shows that gold-silica-gold nanoshell can generate a strong surface plasmon resonance[9]. Halas et al have studied the optical properties of concentric nanoshell structures using the plasmon hybridization theory, and suggested the complex structure of nanoshell can be viewed as a sum of the contributions of the dipolar and higher order hybrid multipolar plasmon modes[10], which provides a new approach to well analyze the optical properties of nanoshell. Debelaa et al have reported surface plasmon resonances in ellipsoidal bimetallic nanoparticles using the Laplace equation, and the results show that the LSPR depends on surrounding dielectric function[11]. Wu et al have studied optical properties of bimetallic gold and silver alloy nanoshell for biomedical applications in vivo with Mie scattering theory and plasmon hybridization theory, and the result indicates that the lower energy mode has a distinct red-shift with increasing core radius[12]. Schiditabar et al have investigated the resonance light scattering properties of silver-dielectric-silver nanoshell based on quasi-static approach, the results show that LSPR are optimized by tuning the geometrical dimensions[13]. The light absorption spectra properties of a bimetallic multilayer nanoshell (silver-dielectric-silver, gold-dielectric-silver and silver-dielectric-gold), the results show that relatively small thickness of outer silver shell and large dielectric constants of middle dielectric layer of gold-dielectric-silver nanoshell are suitable to obtain the triple plasmon resonance[14].

Recently, the optical properties of nanotube have also been widely investigated. Bahari et al have studied metalair-metal cylinder theoretically, and given the effect of increasing the interwall spacing between two metals on plasmon dispersion relation[15]. Zhu et al have investigated the effect of local dielectric environment on the resonance light scattering of $\mathrm{Au}-\mathrm{Ag}$ bimetallic nanotube, the results present a potential for design and fabrication of optical refractive index nanosensors based on light scattering[16]. Gao et al have investigated the geometrical parameters controlled focusing and enhancing near field in infinite circular silver-dielectric-silver cylinder

\footnotetext{
* Corresponding Author: mayewan@126.com
} 
with quasi-static approach[17]. A stronger local field enhancement can be obtained in the middle dielectric wall between inner silver and outer tube at the resonance wavelength[18]. Moradi has theoretically studied how the plasmon excitations of the system depend on the dielectric difference between the core and shell in coated metallic nanowires[19]. Zhu et al have reported the local dielectric environment dependent local field enhancement properties in double concentric silver nanotube using plasmon hybridization method and quasi-static calculation[20]. Daneshfar et al have studied optical and spectral tunability of multilayer spherical and cylindrical nanoshell with quasi-static theory[21]. Wu have studied the the refractive sensitivity of silver-dielectric-silver and gold-dielectricgold nanotube, and the result shows that the sensing ability can be improved by reducing the thickness of the inner gold shell or increasing the thickness of the outer gold shell[22, 23].

Most of previous studies have mainly focused on gold-dielectric-gold nanotube, where the core and outer shell have the same nobel metal, while the core and outer shell with different nobel metals are less reported. In this paper, we investigate the influences of inner core radius, the dielectric functions of inner core and surrounding medium on gold-dielectric-silver nanotubes in detail, and the distributions of local electric field factor are also reported.

\section{MODEL AND THEORETICAL METHOD}

In this paper, we consider optical property of gold-dielectric-silver nanotube. The geometry of infinite threelayered gold-dielectric-silver nanotube is given in Fig.1. The geometry consists of a infinite length gold nanowire core with a radius $r_{1}$ and dielectric function $\varepsilon_{1}$, the outer silver nanotube with a thickness $r_{3}-r_{2}$ and dielectric function $\varepsilon_{3}$. The core and nanotube are separated by a middle dielectric layer with a thickness $r_{2}-r_{1}$ and dielectric function $\varepsilon_{2}$, and the gold-dielectric-silver nanotube is suspended in the embedding medium with dielectric function $\varepsilon_{4}$. The total diameter of gold-dielectric-silver nanotube is much smaller than the incident wavelength, the surface charges in gold-dielectric-silver nanotube oscillate like a simple electric dipole and then the quasistatic approximation can be used to calculate the optical property of absorption cross section[24]. The diameter of gold-dielectric-silver nanotube is much smaller than the incident wavelength, thus the induced field in the region outerside the shell is the same as a dipole. The absorption cross section can be gotten from polarizability by using the scattering theory $C_{a b s}=k \operatorname{Im}(\alpha)[25,26]$, where $\alpha$ is the polarizability.

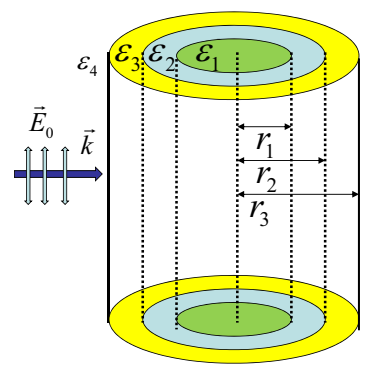

FIG. 1: The geometry of gold-dielectric-silver nanotube

In Drude model[27], the frequency-dependent inner gold core $\varepsilon_{1}$ and outer silver shell $\varepsilon_{3}$ have real and imaginary components. The dielectric functions of gold and silver could be written as follows

$$
\varepsilon(\omega)=\varepsilon_{\text {exp }}(\omega)+\frac{\omega_{p}{ }^{2}}{\omega^{2}+i \omega \gamma_{b u l k}}-\frac{\omega_{p}{ }^{2}}{\omega^{2}+i \omega\left(\gamma_{b u l k}+\frac{V_{F}}{R}\right)}
$$

where $\varepsilon_{\exp }(\omega)$ is the experimental bulk dielectric function[28]. $\omega_{p}=1.37 \times 10^{16} \mathrm{~Hz}$ denotes the plasma frequency of bulk gold and $\omega_{p}=1.364 \times 10^{16} \mathrm{~Hz}$ for silver, $\gamma_{b u l k}=1.07 \times 10^{13} \mathrm{~Hz}$ is electron collision damping in the gold and $\gamma_{\text {bulk }}=3.23 \times 10^{13} \mathrm{~Hz}$ for silver. $V_{F}=1.4 \times 10^{6} \mathrm{~m} / \mathrm{s}$ is the Fermi velocity, $R=r_{3}-r_{2}$ for gold nanotube and $R=2 r_{1}$ for inner silver core. 


\section{RESULTS AND DISCUSSION}

According to the plasmon hybridization theory[9], gold-dielectric-silver nanotube can be considered as a plasmon interaction between the plasmon response of a gold wire with its energy mode $\left|\omega_{s}\right\rangle$ and outer silver nanotube $\left(\left|\omega_{-}\right\rangle\right.$and $\left.\left|\omega_{+}\right\rangle\right)$, which results in four new plasmon $\left|\omega_{+}^{+}\right\rangle,\left|\omega_{+}^{-}\right\rangle,\left|\omega_{-}^{+}\right\rangle$and $\left|\omega_{-}^{-}\right\rangle$. The coupling between inner gold wire and outer nanotube is given in Fig.2. The higher energy $\left|\omega_{+}^{+}\right\rangle\left(\left|\omega_{-}^{+}\right\rangle\right)$mode corresponds to anti-symmetric. (symmetric) coupling between anti-symmetric plasmon mode $\left|\omega_{+}\right\rangle$and the inner gold wire plasmon $\left|\omega_{s}\right\rangle$. And the lower energy $\left|\omega_{-}^{-}\right\rangle\left(\left|\omega_{+}^{-}\right\rangle\right)$mode corresponds to anti-symmetric (symmetric) coupling between symmetric plasmon mode $\left|\omega_{-}\right\rangle$and $\left|\omega_{s}\right\rangle .\left|\omega_{+}^{+}\right\rangle$mode is usually too weak to be observed in experiments due to the induced charges of inner wire plasmon oppositely aligned with that of outer nanotube plasmon, which weakens the dipole moment. As shown in Fig.2(a), one can find that $\left|\omega_{-}\right\rangle$plays a more role to $\left|\omega_{-}^{-}\right\rangle$than that $\left|\omega_{s}\right\rangle$, where the higher energy $\left|\omega_{-}^{+}\right\rangle$is dominated by $\left|\omega_{s}\right\rangle$. In order fully understand the physical mechanism of the plasmon coupling, Fig. 2(b,c,d) gives the local field intensity distribution of gold-dielectric-silver nanotubes at each LSPR wavelength $\lambda=318 \mathrm{~nm}, \lambda=484 \mathrm{~nm}$ and $\lambda=750 \mathrm{~nm}$, respectively. As shown in Fig.2(b), the wavelength is $\lambda=318 \mathrm{~nm}$, the intense local field appears in middle dielectric layer and outer silver nanotube, where the electric field in inner gold wire and surrounding medium is very weak. The local electric field distribution indicates that the opposite charges signed on the surface of inner dielectric layer and outer nanotube, which will gets strong attractive force[29], thus we think $\lambda=318 \mathrm{~nm}$ is the $\left|\omega_{+}^{-}\right\rangle$mode. Figure 2(c) shows the intense electric field appears in the region of inner gold wire and outer silver nanotube at LSPR wavelengh $\lambda=484 \mathrm{~nm}$, wheres the electric field in the inner gold wire is much stronger, while the electric field is very weak in the middle dielectric layer. The induced charges is opposite on the surface of inner gold wire, while the same sign of induced charges distribute on the inner and outer surface of outer silver nanotube, thus $\lambda=484 \mathrm{~nm}$ is $\left|\omega_{-}^{+}\right\rangle$mode. In addition, one can find that the intense local electric field enhancement appears in the middle dielectric layer due to the opposite charges on the inner and outer surface of middle dielectric layer in Fig.2(d) at LSPR wavelength $\lambda=750 \mathrm{~nm}$, therefore, $\lambda=750 \mathrm{~nm}$ is $\left|\omega_{-}^{-}\right\rangle$ mode. Normally, the lower energies of $\left|\omega_{-}^{-}\right\rangle$mode and $\left|\omega_{-}^{+}\right\rangle$mode can be clearly visible in the infrared region. In this paper, the optical properties of $\left|\omega_{-}^{+}\right\rangle$mode and $\left|\omega_{-}^{-}\right\rangle$mode are studied in detail.
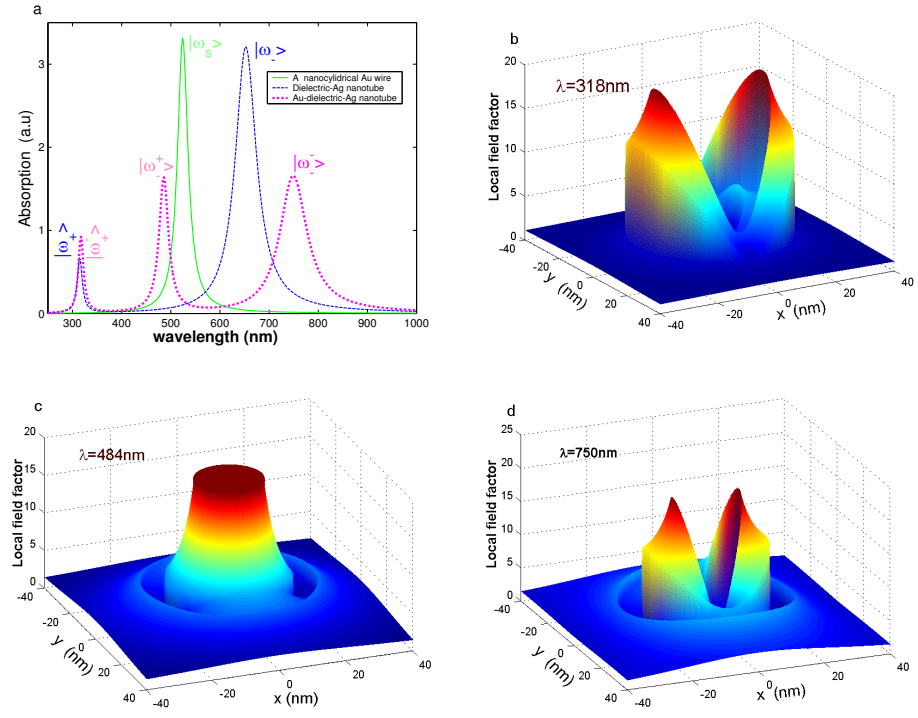

FIG. 2: (a) Absorption spectra of gold-dielectric-silver nanotubes calculated for a single cylindrical gold wire, dielectric-silver nanotube and gold-dielectric-silver nanotubes, $(b, c, d)$ the local electric field intensity distribution of gold-dielectric-silver nanotubes at each LSPR wavelength $\lambda=318 \mathrm{~nm}, \lambda=484 \mathrm{~nm}$ and $\lambda=750 \mathrm{~nm}$, respectively. 
A. The effect of inner core radius with different middle dielectric functions on LSPR of gold-dielectric-silver nanotube

The absorption spectra of gold-dielectric-silver nanotube with different inner core radii and middle dielectric functions are plotted in Fig.3, where the radii of middle dielectric layer $r_{2}$ and the outer silver nanotube $r_{3}$ are fixed at $15 \mathrm{~nm}$ and $25 \mathrm{~nm}$, respectively. The gold-dielectric-silver nanotube is suspended in surrounding medium $\varepsilon_{4}=4.0$. The results show that the intensity, LSPR position and LSPR peak number are sensitive to inner core radius and middle dielectric functions. $\left|\omega_{-}^{-}\right\rangle$mode shows a distinct red-shift with increasing inner core radius from $r_{1}=2 \mathrm{~nm}$ to $r_{1}=12 \mathrm{~nm}$, while $\left|\omega_{-}^{+}\right\rangle$mode shows a little blue-shift. One can find that $\left|\omega_{-}^{-}\right\rangle$mode has a red-shift $\Delta \lambda=198 \mathrm{~nm}$ from $\lambda=652 \mathrm{~nm}$ to $\lambda=854 \mathrm{~nm}$ with $\varepsilon_{2}=2.0$ in Fig.3(a), while its intensity reduces and fades down quickly, these results are agree well with previous studies[13, 14, 18, 20, 22]. The nanotube plasmon of $\left|\omega_{-}\right\rangle$ and $\left|\omega_{+}\right\rangle$could be considered as a constant when the radii of $r_{2}$ and $r_{3}$ are fixed, and in this case the LSPRs are mainly affected by that of inner gold wire $\left|\omega_{s}\right\rangle$ mode[9]. As the inner core radius increases, the separation between inner core and nanotube reduces, and then the coupling between inner core and nanoshell is enhanced. Therefore, the energy of $\left|\omega_{-}^{-}\right\rangle$mode reduces (a red-shift) and $\left|\omega_{-}^{+}\right\rangle$mode enhances (a blue-shift). However, the intensity of $\left|\omega_{-}^{+}\right\rangle$mode show contrary behavior to that of $\left|\omega_{-}^{-}\right\rangle$mode, $\left|\omega_{-}^{+}\right\rangle$mode intensity enhances with increasing inner core radius. The physical mechanism of these properties could be explained as, the intensity of $\left|\omega_{-}^{+}\right\rangle$mode is mainly dominated by inner gold wire $\left|\omega_{s}\right\rangle$. There are more electrons to oscillate with increasing inner core radius and then the intensity of $\left|\omega_{-}^{+}\right\rangle$mode enhanced with increasing inner core radius. It is also found that both the intensity and the peak position of LSPR are also sensitive to middle dielectric function. Compared with Fig.3(a),(b) and (c), the shift of $\Delta \lambda$ is red-shift and the intensity is reduced quickly with increasing $\varepsilon_{2}=2.0$ to $\varepsilon_{2}=6.0$. The induced charges is reduced with increasing middle dielectric function[29], and then $\left|\omega_{-}^{-}\right\rangle$mode shows a red-shift and its intensity is also reduced. In addition, one can find that $\left|\omega_{+}^{-}\right\rangle$mode becomes to appear when inner gold wire radius is bigger enough $\left(r_{1} \geq 4 \mathrm{~nm}\right)$, and its intensity enhances with increasing inner gold wire radius. In summary, LSPR numbers and its intensity can be well tuned by varying the inner gold wire radius and middle dielectric function.
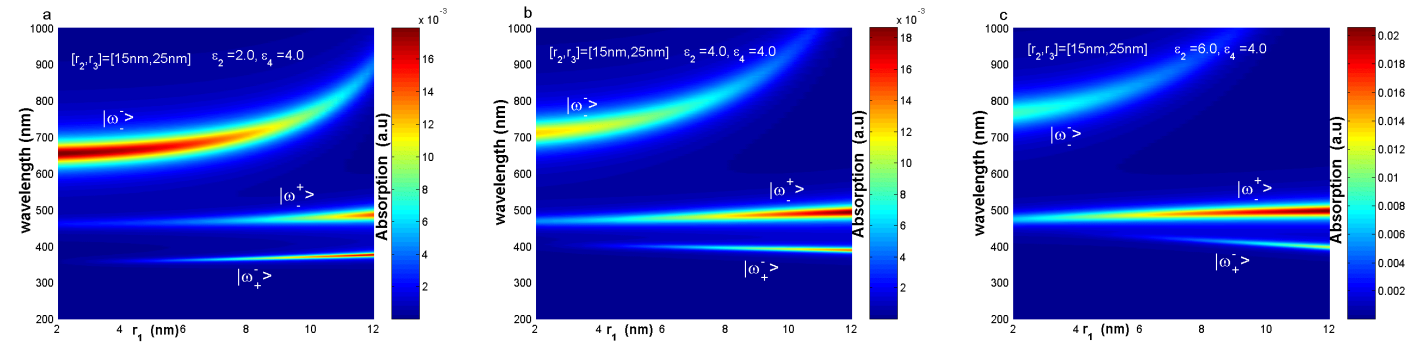

FIG. 3: The effects of inner gold wire radius $r_{1}$ on absorption spectra of gold-dielectric-silver nanotube with $\left[r_{2}, r_{3}\right]=$ $[15 \mathrm{~nm}, 25 \mathrm{~nm}]$ and $\varepsilon_{4}=4.0$. (a) $\varepsilon_{2}=2.0$, (b) $\varepsilon_{2}=4.0$ and (c) $\varepsilon_{2}=6.0$, respectively.
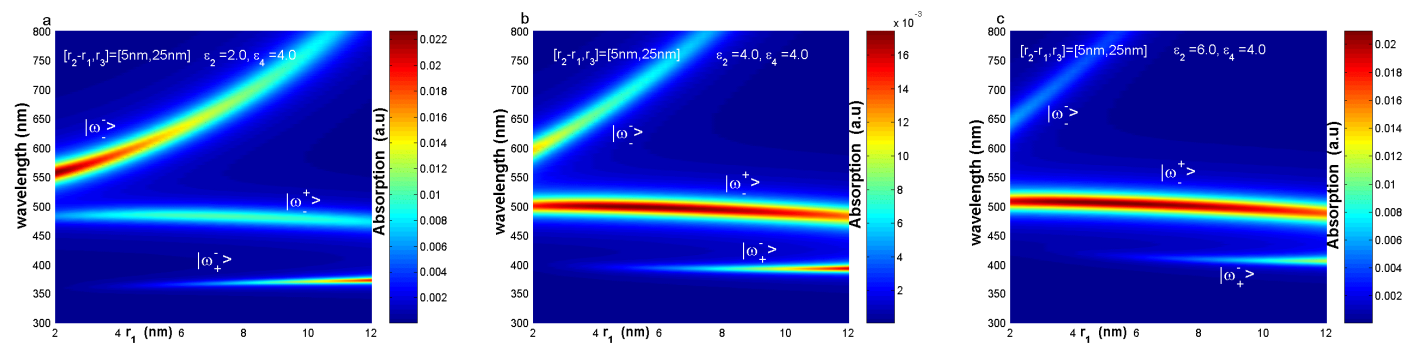

FIG. 4: The effects of inner gold wire radius $r_{1}$ on absorption spectra of gold-dielectric-silver nanotube with $\left[r_{2}-r_{1}, r_{3}\right]=$ [5nm, 25nm] and $\varepsilon_{4}=4.0$. (a) $\varepsilon_{2}=2.0$, (b) $\varepsilon_{2}=4.0$ and (c) $\varepsilon_{2}=6.0$, respectively.

To fully understand the influence of inner gold wire radius on LSPR, the absorption spectra of LSPR are given in Fig.4 with middle dielectric thickness fixed at $r_{2}-r_{1}=5 \mathrm{~nm}$, where the inner gold core radius $r_{1}$ is varied 
from $r_{1}=2 \mathrm{~nm}$ to $r_{1}=12 \mathrm{~nm}$ and the outer nanotube radius is fixed at $r_{3}=25 \mathrm{~nm}$. Compared with Fig.3(a,b,c), the results show that $\left|\omega_{-}^{-}\right\rangle$mode shows a red-shift much more quickly than that of Fig.3(a,b,c) with increasing inner gold core radius, and its intensity also reduces rapidly[17]. $\left|\omega_{-}^{+}\right\rangle$mode shows a little blue-shift, its intensity enhances firstly and then reduces. The physical mechanism could be attributed to plasmon hybridization, the coupling of outer nanotube is effected by the ratio of $r_{2} / r_{3}$ [19]. The coupling is enhanced with increasing the ratio $r_{2} / r_{3}\left(r_{2} / r_{3}=0.28\right.$ for $r_{2}=7 n m$ and $r_{2} / r_{3}=0.68$ for $\left.r_{2}=17 n m\right)$, and then $\left|\omega_{-}^{-}\right\rangle$mode shows a red-shift and $\left|\omega_{-}^{+}\right\rangle$mode has a blue-shift. An increase in middle dielectric layer will reduces outer nanotube thickness, the increasing middle dielectric radius leads to interface curvatures of the middle dielectric thickness reduces, and then the surface charges concentration at both inner gold wire and outer silver nanotube of the middle dielectric thickness reduces. Thus the surface charges induced electrostatic attraction leads to the local electric field intensity reduces. On the other hand, there are less effective frees to oscillate, and then the energy of $\left|\omega_{-}^{-}\right\rangle$mode and $\left|\omega_{-}^{+}\right\rangle$ mode are also reduced. It means that the intensity of LSPR is controlled by two factors, one is the coupling, and the other is the induced charges. In addition, compared with Fig.4(a,b,c), one can find that the LSPR of $\left|\omega_{-}^{-}\right\rangle$mode shows red-shift quickly with increasing middle dielectric function and its intensity fades down quickly[29]. The third peak $\left|\omega_{+}^{-}\right\rangle$mode becomes to appear when $r_{1}$ is bigger enough, i.e $r_{1}=5 \mathrm{~nm}$, whose optical property is similar to that of Fig.3.

\section{B. The effect of middle dielectric layer radius on LSPR of gold-dielectric-silver nanotube}

Figure 5 shows the effects of the middle dielectric radius varied from $r_{2}=8 \mathrm{~nm}$ to $r_{2}=23 \mathrm{~nm}$ on LSPR, where the radii of inner gold wire $r_{1}$ and the outer silver nanotube $r_{3}$ are fixed at $5 \mathrm{~nm}$ and $25 \mathrm{~nm}$. Figure.5a shows that the $\left|\omega_{-}^{-}\right\rangle$mode takes place a blue-shift firstly and then a red-shift rapidly with increasing middle dielectric radius, its intensity enhancement only appears when the middle dielectric layer has a thin thickness (about $r_{2}=12 \mathrm{~nm}$ ) and then fades down rapidly as the thickness of middle dielectric layer is further increased. It is known that the plasmon coupling is controlled by the thickness of nanotube according to the plasmon hybridization theory. The plasmon responses of a gold wire with its energy mode $\left|\omega_{s}\right\rangle$ could be considered as a constant when the inner gold wire radius is fixed. Thus the LSPR is mainly effected by the outer nanotube $\left(\left|\omega_{+}\right\rangle\right.$and $\left.\left|\omega_{-}\right\rangle\right)[19,22,23]$. The radius ratios $\left(r_{2} / r_{3}\right)$ is increased from $r_{2} / r_{3}=0.67$ for $r_{2}=10 \mathrm{~nm}$ to $r_{2} / r_{3}=0.8$ for $r_{2}=20 \mathrm{~nm}$, and then the coupling of nanotube is enhanced. Therefore, $\left|\omega_{-}\right\rangle$mode is reduced and $\left|\omega_{+}\right\rangle$mode is increased. $\left|\omega_{-}^{-}\right\rangle$mode shows a red-shift and $\left|\omega_{-}^{+}\right\rangle$mode shows a blue-shift. On the other hand, the thickness of middle dielectric layer is also increased with increasing middle layer radius, and then the separation between the inner gold wire and outer nanotube is increased. The attractive strengthen between the inner silver wire and outer nanotube will be reduced, thus the LSPR peak of $\left|\omega_{-}^{-}\right\rangle$mode shows a red-shift. In addition, the intensity of LSPR increases firstly and then reduces gradually with increasing the inner core radius. This behavior could be explained as, although the coupling is stronger with thin thickness, there are less electrons to participant in oscillating when thickness become much thinner, thus the resonance become decrease and absorption gets less intensity. In addition, one can find that an increase in middle dielectric function will leads to the LSPR of $\left|\omega_{-}^{-}\right\rangle$mode a red-shift and its intensity fades down quickly[29]. The optical property of $\left|\omega_{+}^{-}\right\rangle$mode is contrary to that of Fig.3 and Fig.4, its intensity fades down and becomes to disappear with increasing middle dielectric layer radius $r_{2}$, this phenomenon is agree with previous study[20]. The effect of middle dielectric function $\varepsilon_{2}$ on $\left|\omega_{+}^{-}\right\rangle$mode is similar to that of Fig.3 and Fig.4.
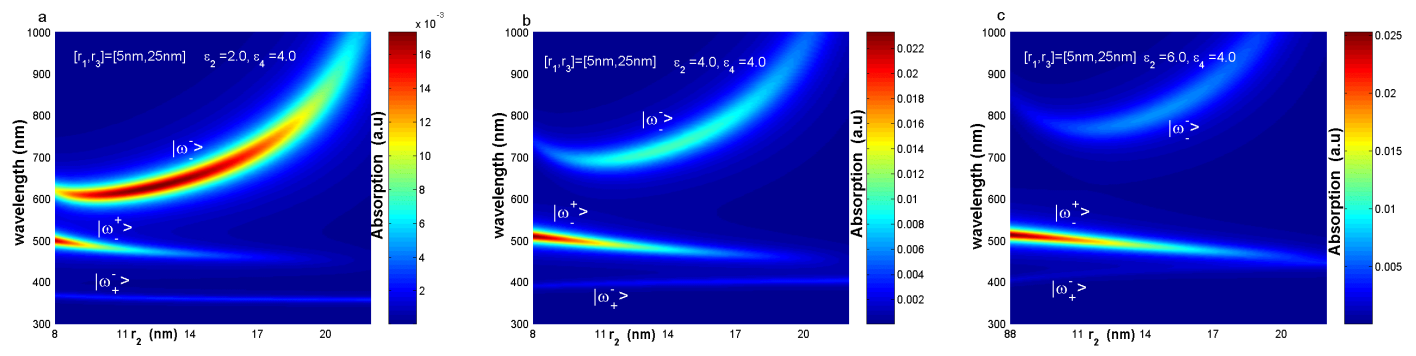

FIG. 5: The effects of middle dielectric radius $r_{2}$ on absorption spectra of gold-dielectric-silver nanotube with $\left[r_{1}, r_{3}\right]=$ [5nm, 25nm and $\varepsilon_{4}=4.0$. (a) $\varepsilon_{2}=2.0$, (b) $\varepsilon_{2}=4.0$ and (c) $\varepsilon_{2}=6.0$, respectively. 

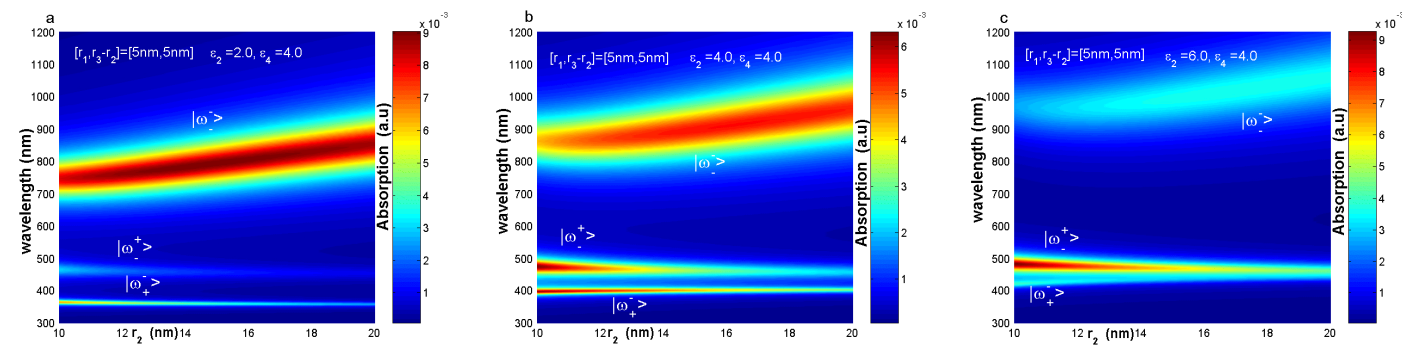

FIG. 6: The effects of middle dielectric radius $r_{2}$ on absorption spectra of gold-dielectric-silver nanotube with $\left[r_{1}, r_{3}-r_{2}\right]=$ [5nm, 5nm] and $\varepsilon_{4}=4.0$. (a) $\varepsilon_{2}=2.0$, (b) $\varepsilon_{2}=4.0$ and (c) $\varepsilon_{2}=6.0$, respectively.

Our next goal is to study the effect of outer middle dielectric thickness on LSPR, where the inner gold wire radius $r_{1}$ and outer nanotube thickness $r_{3}-r_{2}$ are fixed at $r_{1}=5 \mathrm{~nm}$ and $r_{3}-r_{2}=5 \mathrm{~nm}$, while the middle dielectric radius $r_{2}$ is varied from $r_{2}=10 \mathrm{~nm}$ to $r_{2}=20 \mathrm{~nm}$, which is given in Fig.6. Figure 6 shows that $\left|\omega_{-}^{-}\right\rangle$mode shows a red-shift and $\left|\omega_{-}^{+}\right\rangle$mode has a little blue-shift with increasing middle dielectric radius $r_{2}$. The coupling of nanotube $\left(\left|\omega_{-}\right\rangle\right.$mode and $\left|\omega_{+}\right\rangle$mode) is enhanced with increasing the ratio of $r_{2} / r_{3}[9]$. On the other hand, the total size of gold-dielectric-silver nanotube is also increased, thus the phase retardation will leads to reduce the intensity of LSPR, and then both $\left|\omega_{-}^{-}\right\rangle$mode and $\left|\omega_{-}^{+}\right\rangle$are reduced[12], thus $\left|\omega_{-}^{-}\right\rangle$mode shows a red-shift. It means that $\left|\omega_{-}^{-}\right\rangle$mode is controlled by two factors, one is the coupling between gold wire and silver nanotube, the other is the phase retardation. $\left|\omega_{-}^{-}\right\rangle$mode shows a blue-shift when the coupling plays a dominate role than phase retardation, otherwise, $\left|\omega_{-}^{-}\right\rangle$mode shows a red-shift. Figure 6 means that phase retardation plays a dominate role than the coupling, and then $\left|\omega_{-}^{-}\right\rangle$mode shows a red-shift. In addition, the intensity reduces with increasing middle dielectric function, which is similar to that of Fig.5.

\section{The effect of outer silver radius on LSPR of gold-dielectric-silver nanotube}
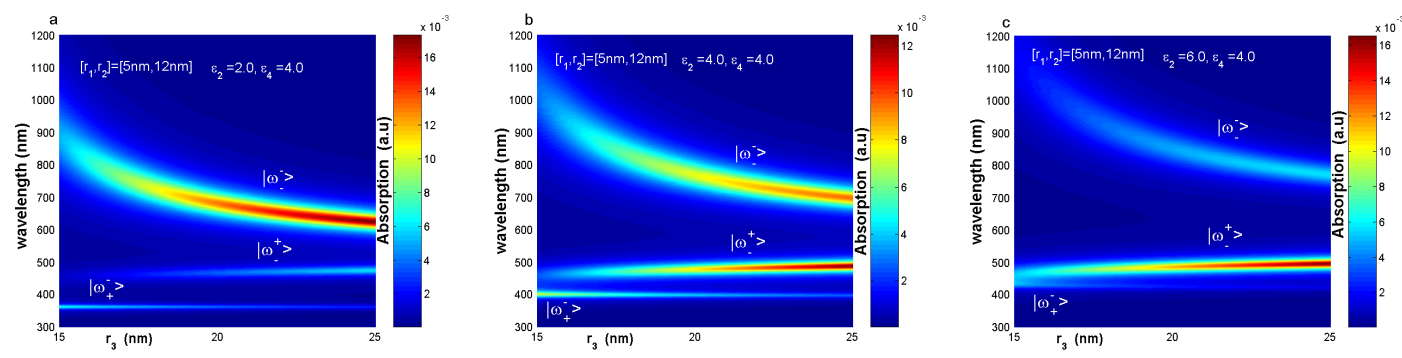

FIG. 7: The effects of outer nanotube radius $r_{3}$ on absorption spectra of gold-dielectric-silver nanotube with $\left[r_{1}, r_{2}\right]=$ [5nm, 10nm $]$ and $\varepsilon_{4}=4.0$. (a) $\varepsilon_{2}=2.0$, (b) $\varepsilon_{2}=4.0$ and (c) $\varepsilon_{2}=6.0$, respectively.

We further investigate the effects of outer nanotube radius $r_{3}$ on LSPRs, the results are shown in Fig.7, where the radii of inner core $r_{1}$ and the middle dielectric layer $r_{2}$ are fixed at $5 \mathrm{~nm}$ and $12 \mathrm{~nm}$, while the outer radius $r_{3}$ is varied from $r_{3}=15 \mathrm{~nm}$ to $r_{3}=25 \mathrm{~nm}$. One can find that the $\left|\omega_{-}^{-}\right\rangle$mode has a blue-shift distinctly with increasing the outer radius from $r_{3}=15 \mathrm{~nm}$ to $r_{3}=25 \mathrm{~nm}$, and the intensity of LSPR is enhanced. $\left|\omega_{-}^{+}\right\rangle$mode is a little red-shift. The ratio of $r_{2} / r_{3}$ is reduced with increasing outer nanotube radius $r_{3}$, and then $\omega_{+}$reduces and $\omega_{-}$ enhances[20,22]. Thus the coupling between the gold wire and nanotube is reduced, in this case the intensity of $\left|\omega_{-}^{-}\right\rangle$mode is increased and $\left|\omega_{-}^{+}\right\rangle$mode is reduced. On the other hand, the total size is also increased with increasing outer nanotube radius $r_{3}$, which reduces the LSPR intensity due to phase retardation effect, thus both $\left|\omega_{-}^{-}\right\rangle$mode and $\left|\omega_{-}^{+}\right\rangle$are reduced. Figure 7 shows that the coupling is more dominate than that of phase retardation. It is also find that the absorption gets more intensity with increasing outer shell thickness due to more electrons to participant in oscillating when thickness become much thicker. In addition, Fig.7 shows the intensity is reduced with increasing middle dielectric function[22]. Figure 8 shows the effects of the ratio of $r_{1} / r_{2} / r_{3}=$ constant on 

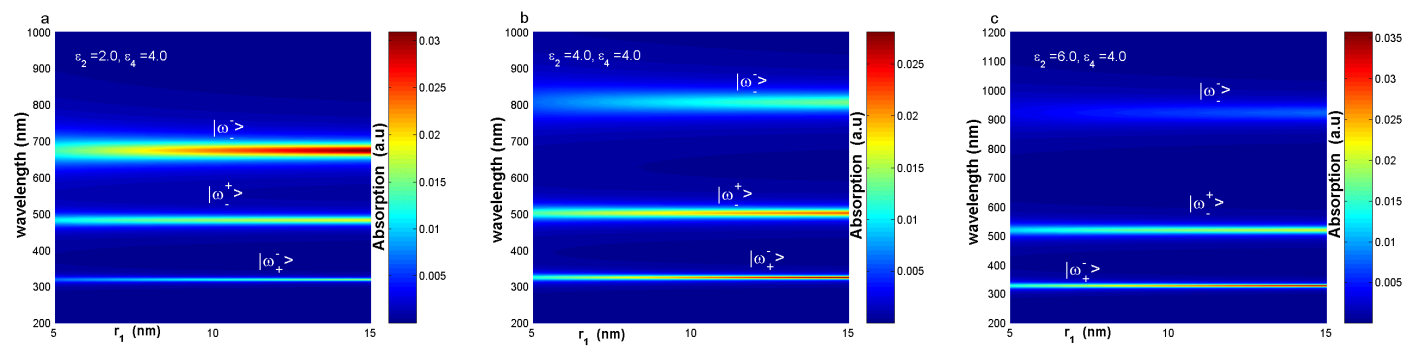

FIG. 8: The effects of the ratio of $r_{1} / r_{2} / r_{3}=$ constant on absorption spectra of gold-dielectric-silver nanotube with $r_{1}=5 \mathrm{~nm}$ and $\varepsilon_{4}=4.0$. (a) $\varepsilon_{2}=2.0$, (b) $\varepsilon_{2}=4.0$ and (c) $\varepsilon_{2}=6.0$, respectively.

LSPR, the results show that the peak is no-shift and its intensity enhances with increasing the inner gold wire radius, which is agree well with theoretical studies[19], where the LSPR shows no-shift when $r_{1} / r_{2}=$ constant, $r_{2} / r_{3}=$ constant and $r_{1} / r_{3}=$ constant .

\section{The effect of surrounding medium dielectric function on LSPR of gold-dielectric-silver nanotube}

In order to understand the absorption spectra of gold-dielectric-silver nanotube, we also gain an insight into the effects of surrounding medium dielectric functions $\varepsilon_{4}$ on LSPR in Fig.9. Figure 9(a) shows that the LSPR of $\left|\omega_{-}^{-}\right\rangle$ mode is sensitive to the surrounding medium $\varepsilon_{4}$, which shows a distinct red-shift and its intensity enhances quickly with increasing the surrounding medium $\varepsilon_{4}$, while the LSPR of $\left|\omega_{-}^{+}\right\rangle$mode is not sensitive to the surrounding medium $\varepsilon_{4}$, the LSPR position almost keeps at a constant and its intensity fades down. In the reports of Deeb[30] and $\mathrm{Zhu}[31]$, both the induced surface charges and the absorption intensities are related to the electric field discontinuity at the particle interface. According to plasmon hybridization theory, the inside and outside surface of middle dielectric layer have the opposite induced surface charges (positive and negative charges) in $\left|\omega_{-}^{-}\right\rangle$mode. And then the local field in the dielectric layer is polarized in the direction of the depolarization field around the surrounding media. The attractive force between the inside and outside of the middle dielectric layer leads to an increase in the induced charges density of the dielectric layer, and then the absorption is increased. Therefore, $\left|\omega_{-}^{-}\right\rangle$mode is sensitive to the surrounding medium function $\varepsilon_{4}$. However, in $\left|\omega_{-}^{+}\right\rangle$mode, the surfaces of the dielectric layer (inside and outside) have the same sign charge, the local field in the dielectric layer is perpendicular to the depolarization field around the surrounding media, and then the surface density of induced charges is proportional to the change of the local electric field inside and outside of the particle. Therefore, the absorption intensity of $\left|\omega_{-}^{+}\right\rangle$mode mode is not very sensitive to the surrounding dielectric functions. The intensity difference between $\left|\omega_{-}^{-}\right\rangle$mode and $\left|\omega_{-}^{+}\right\rangle$ mode is increased with increasing the surrounding media functions $\varepsilon_{4}$. In other words, the intensity difference is sensitive to the surrounding media functions $\varepsilon_{4}$. Recent studies have shown that the plasmon absorption intensity difference between $\left|\omega_{-}^{-}\right\rangle$mode and $\left|\omega_{-}^{+}\right\rangle$mode has been used for refractive index sensitivity[3, 6, 22, 31, 32], the effect of the radii of inner core and middle dielectric thickness, and middle dielectric function on LSPR are also studied, which are given in Fig.9(b,c,d). Compared with Fig.9(a), Fig.9(b) shows that the intensity of $\left|\omega_{-}^{-}\right\rangle$ mode enhances quickly with increasing inner gold wire radius, while the intensity of $\left|\omega_{-}^{+}\right\rangle$mode almost remains at a constant, which is contrary to that of sphere nanoshll[32]. As increasing surrounding medium function, the intensity difference between $\left|\omega_{-}^{-}\right\rangle$mode and $\left|\omega_{-}^{+}\right\rangle$mode is slower than that of Fig.9(a) with smaller inner radius. In other words, the thinner gold wire radius is more superior than that of thicker gold wire to be used in refractive index sensitivity. Compared with Fig.9(a) and (b), Fig.9(c) shows that the intensity of $\left|\omega_{-}^{+}\right\rangle$mode enhances slower with thinner thickness of outer nanotube than that of Fig.9(a) and (b) with thicker thickness of outer nanotube. In addition, the full widths at half maximum is increased. Finally, the effect of middle dielectric function is also investigated. Figure 9(d) shows that the LSPR of $\left|\omega_{-}^{+}\right\rangle$mode is mainly effected by the middle dielectric function $\varepsilon_{2}$, the intensity of $\left|\omega_{-}^{+}\right\rangle$mode enhances quickly and the full widths at half maximum is increased when the middle dielectric function $\varepsilon_{2}$ is increased. In this case, the intensity difference is reduced, which is not suitable for refractive index sensitivity. In summary, a higher refractive index sensitivity can be improved by thinner core radius $r_{1}$, thicker outer nanoshell (smaller middle layer radius $r_{2}$ or bigger outer shell radius $r_{3}$ ) and smaller middle layer dielectric functions $\varepsilon_{2}$. 

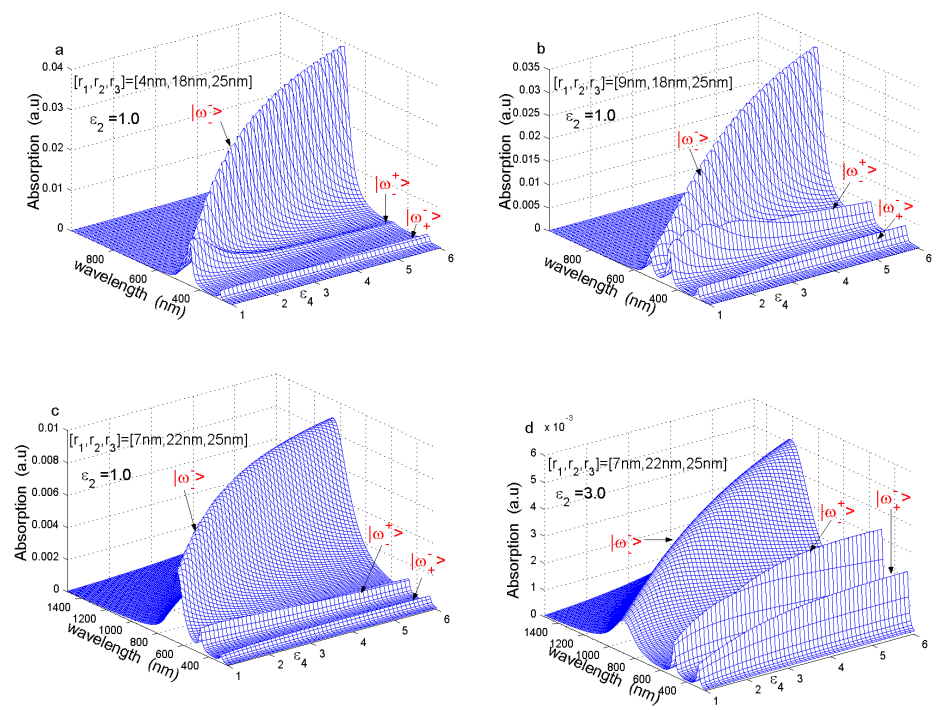

FIG. 9: The effects of surrounding medium $\varepsilon_{4}$ on LSPR of gold-dielectric-silver nanotube. (a) $\left[r_{1}, r_{2}, r_{3}\right]=[4 \mathrm{~nm}, 18 \mathrm{~nm}, 25 \mathrm{~nm}]$ and $\varepsilon_{2}=1.0$, (b) $\left[r_{1}, r_{2}, r_{3}\right]=[9 \mathrm{~nm}, 18 \mathrm{~nm}, 25 \mathrm{~nm}]$ and $\varepsilon_{2}=1.0$, (c) $\left[r_{1}, r_{2}, r_{3}\right]=[7 \mathrm{~nm}, 22 \mathrm{~nm}, 25 \mathrm{~nm}]$ and $\varepsilon_{2}=1.0$, and (d) $\left[r_{1}, r_{2}, r_{3}\right]=[7 \mathrm{~nm}, 22 \mathrm{~nm}, 25 \mathrm{~nm}]$ and $\varepsilon_{2}=3.0$.

\section{E. The local electric field distribution of gold-dielectric-silver nanotube}

In order to find the physical mechanism of gold-dielectric-silver nanotube, we calculate the distributions of local electric field intensity at the resonance wavelength corresponding to $\left|\omega_{-}^{-}\right\rangle$mode with different inner gold wire radius $r_{1}$, middle dielectric layer radius $r_{2}$ and middle dielectric functions $\varepsilon_{2}$, which are given in Fig.10. As shown in Fig.10(a), one can find that the electric field intensity is concentrated in the middle dielectric layer, where the inner gold wire and outer nanotube is very weak[15, 17]. Compared with Fig.10(a), Fig.10(b) shows that the local electric field intensity reduces with increasing inner gold wire radius $r_{1}$. The coupling between inner gold wire and outer nanotube is enhanced when the separation between inner gold wire and outer nanotube is reduced with increasing inner gold wire radius $r_{1}$. Therefore, the electric field intensity is concentrated with small inner gold wire in middle dielectric layer, which is agree well with previous studies[17]. By comparing with Fig.10, one can find that the local field intensity reduces quickly as the thickness of middle dielectric layer is increased. Meanwhile, the electric field in the outer nanotube and outer surrounding increases, whereas the electric field becomes weak with increasing the radius of middle dielectric layer. The physical mechanisms of these phenomena can be explained as follows. The gap between the inner core and outer nanotube is increased with an increase in the radius of middle dielectric layer, and then the attraction between the inner gold core and outer nanotube is reduced. In other words, the weakened attraction results in a decrease in the local electric field in middle dielectric layer. In addition, the local electric field intensity in the middle dielectric layer reduces with increasing the middle dielectric function $\varepsilon_{2}[19,20,29]$. The bigger dielectric function will reduce the surface charges, and then the restoring force of the collective oscillation is reduced due to dielectric screening, which results in the decrease of plasmon energy. Therefore, a better enhancement electric field intensity can be obtained with smaller inner gold wire radius and smaller middle dielectric function[29].

\section{CONCLUSIONS}

In conclusion, the optical properties of gold-dielectric-silver nanotubes have been studied using the quasi-static approximation. The results show that LSPR and its LSPR numbers can be well tuned by the radius of inner gold core, the thickness of middle dielectric layer and outer nanotube. $\left|\omega_{-}^{-}\right\rangle$mode shows a distinct red-shift with increasing the thickness of inner inner gold core, middle dielectric layer, while a blue-shift with increasing the thickness of outer nanotube. $\left|\omega_{-}^{-}\right\rangle$mode is sensitive to middle layer dielectric functions and surrounding medium dielectric functions. The intensity difference is enhanced with thinner core radius and smaller middle layer dielectric functions. Higher refractive index sensitivity can be obtained with a smaller radius of inner gold 

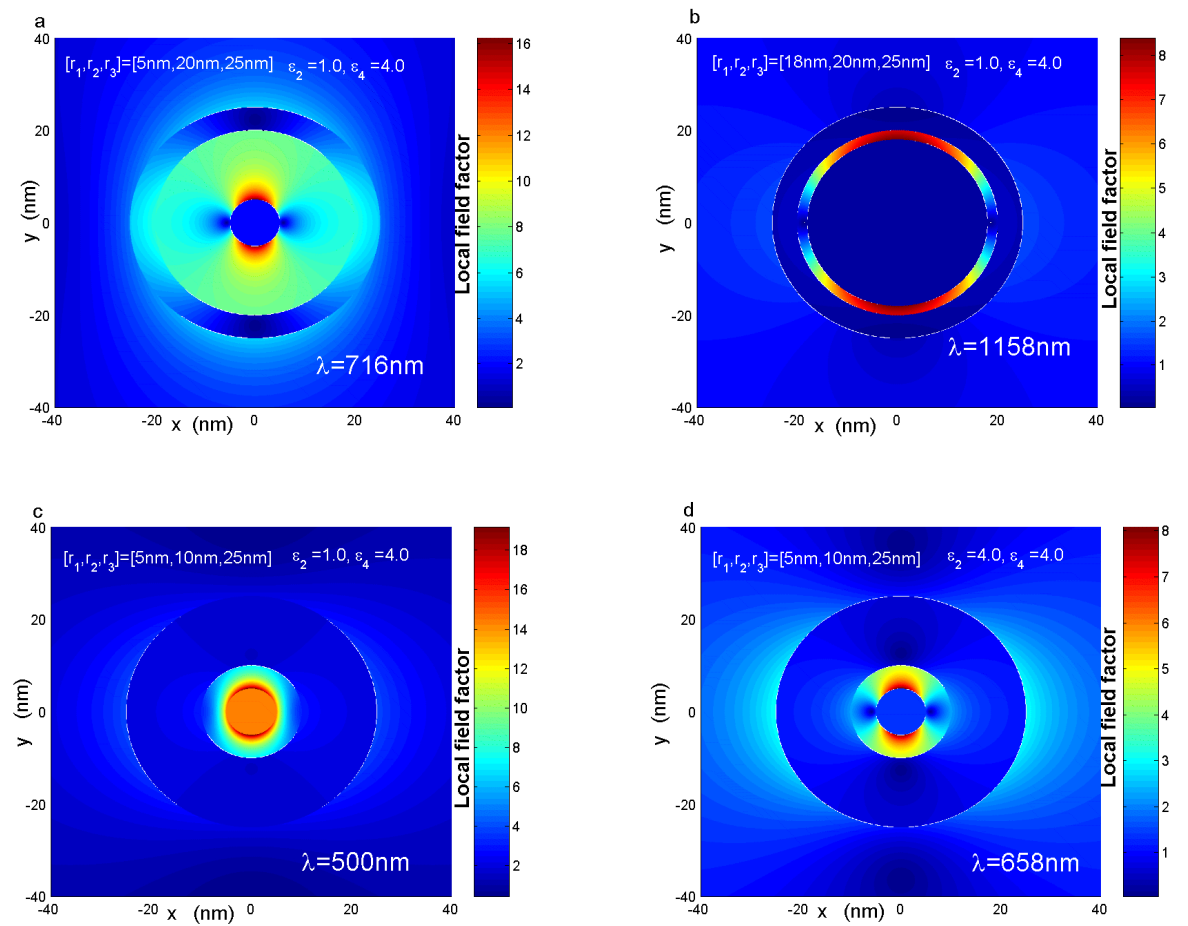

FIG. 10: The distributions of local electrical field intensity of gold-dielectric-silver at the resonance wavelength corresponding to $\left|\omega_{-}^{-}\right\rangle$mode with different middle dielectric radii and middle dielectric functions. (a) $\left[r_{1}, r_{2}\right]=[5 \mathrm{~nm}, 20 \mathrm{~nm}],\left[\varepsilon_{2}, \varepsilon_{4}\right]=$ $[1.0,4.0]$ and $\lambda=716 \mathrm{~nm}$, (b) $\left[r_{1}, r_{2}\right]=[18 \mathrm{~nm}, 20 \mathrm{~nm}],\left[\varepsilon_{2}, \varepsilon_{4}\right]=[1.0,4.0]$ and $\lambda=1158 \mathrm{~nm}$, (c) $\left[r_{1}, r_{2}\right]=[5 \mathrm{~nm}, 10 \mathrm{~nm}],\left[\varepsilon_{2}, \varepsilon_{4}\right]=$ $[1.0,4.0]$ and $\lambda=500 \mathrm{~nm}(\mathrm{~d}) .\left[r_{1}, r_{2}\right]=[5 \mathrm{~nm}, 10 \mathrm{~nm}],\left[\varepsilon_{2}, \varepsilon_{4}\right]=[4.0,4.0]$ and $\lambda=658 \mathrm{~nm}$.

core and a smaller middle dielectric function. In addition, the distributions of local electric field intensity are also calculated.

Funding Infromation: This work is supported by the Programs for Anhui Provincial Natural Science Foundation (1808085MA05 and 1808085MA20), Excellent Young Talents in University of Anhui Province (gxyq2017027), the key Scientific Research Foundation of Anhui Provincial Education Department (KJ2019A0564, KJ2018A0366), and Higher educational Quality engineering projects of Anhui Province (2020szsfkc0540, 2020szsfkc0548, 2020jyxm1080, 2018zygc062 and aqnu2019jyzc066).

Competing interests: The authors declare that they have no competing interests.

Author's contributions:Ye-Wan Ma and Zhao-Wang Wu wrote the paper and simulate the simulations. Juan $\mathrm{Li}$, Yan-Yan Jiang, Xun-Chang Yin, Ming-Fang Yi, and Li-Hua Zhang approve the writing and give some methods.

References:

[1] Sharma R; Roopak S; Pathak N.K; Ji A, Sharma R.P. Study of Surface Plasmon Resonances of Core-Shell Nanosphere: A Comparison between Numerical and Analytical Approach. Plasmonics,2017,12,977-986

[2] Nie S; Enmory S.R. Probing Single Molecules and Single Nanoparticles by Surface-Enhanced. Science, 1997, 275,11021106

[3] Mayer K.M; Hafner J.H. Localized surface plasmon resonance sensors. Chem.Rev, 2011,111,3828-3857

[4] Warnes W.L; Dereux A; Bobesen T.W. Surface plasmon subwavelength optics. Nature, 2003,424,824-830

[5] Kelly K.L; Coronado .E; Zhao L.L; Schatz G.C. The optical properties of metal nanoparticles: The influence of size, shape, and dielectric enviroment. J.Phys.Chem.B, 2003, 107,668-677.

[6] Mayer K.M; Hafner J.H. Localized surface plasmon resonance sensors. Chem.Rev,2011,111,3828-3857

[7] Maier S.A. Plasmonics: Fundamentals and Application (2007 Springer)

[8] Xia X.H; Liu Y; Backman; V Ameer G.A. Engineering sub-100nm multi-layer nanoshells. Nanotechnolo$g y, 2006,17,5435-5440$ 
[9] Prodan E; Radbloff C; Halas N.J; Nordander P. A hybridization model for the plasmon response of complex nanostructures. Science, 2003,302,419-422

[10] Radloff C and Halas N.J. plasmonic properties of concentric nanoshells Nano.Lett,2004,4,1323-1327

[11] Debelaa S; Mesfinb B; Senbetab T. surface plasmon resonances in ellipsoidal bemetallic nanoparticles. Photon.Nanostruct:Fundam.Appl,2019,33,571-577.

[12] Wu D.J; Liu X.J. Optimization of the bimetallic gold and silver alloy nanoshell for biomedical applications in vivo. Appl.Phys.Lett ,2010,97,061904-061907

[13] Schirzaditabar F; Saliminasab M. Optimization of silver-dielectric-silver nanoshell for sensing application. Physics of plasmas. 2013,20,082102

[14] Schirzaditabar F; Saliminasab M; Nia B.A. Triple plasmon resonance of bimetal nanoshell. Physics of plasmas. 2014,21,072102

[15] Bahari A; Amraie E. Propagation of surface hybrid modes on metallic cylindrical nanoshells. Physics of plasmas. $2012,19,114502$

[16] Zhu J; Li X; L J.J; Zhao J.W. Enlarge the biologic coating-induced absorbance enhancement of Au-Ag bimetallic nanoshells by tuning the metal composition. Spectrochimica Acta-Part A,2018,189,571-577

[17] Gao S.Y; Li P.B; Li F.L. Geometrical parameters controlled focusing and enhancing near field in infinite circular metaldielectric multilayered cylinder. Appl.Phys.Lett,2013,102,123107

[18] Ovidio P and Pal U. geometrical tunability of linear optical response of silica-gold double concentric nanoshell. J.Phys.Chem.C,2010,114,4414-4417

[19] Moradi A. Plasmon hybridization in coated metallic nanowires. J.Opt.Soc.Am.B, 2012, 29, 625-629

[20] J Zhu; Li J.J; Zhao J.W. Local dielectric environment dependent local electric field enhancement in double concenric silver nanotube. J.Phys.Chem.C, 2013, 117, 584-592

[21] Daneshfar N. Effect of interparticle plasmon coupling and temperature on the optical properties of bimetallic composite nanoparticles with a core-shell structure. J. Appl. Phys,2015,117,123105(1-11)

[22] Wu Z.W; Ma Y.W; Jiang Y.Y; Li J; Yin .X.C; Zhang L.H; Yi M.F. Theoretical study of optimizing the refractive index sensitivity of dielectric-metal nanotube by tuning the geometrical parameters. Optics Communications,2019,449 57-62

[23] Wu Z.W; Ma Y.W; Zhang L.H;Yin .X.C; Zhan S.B. Optical tunability of silver-dielectric-silver multi-layered cylindrical nanotube using quasi-static approximation. Chin.Phys.Lett,2018,35 114201

[24] Haus J.W; Zhou H.S; Takami S; Hirasawa M; Honma I; Komiyama H. Enhanced optical properties of metal-coated nanoparticles. J.Appl.Phys,1993, 739,1043-1048

[25] Jackson J.D. Classical electrodynamics (1998, 3rd edn. Wiley, Hoboked)

[26] Bohren C.F; Huffman D R. Absorption and Scattering of Light by Small Particles (2000, Wiley, New York)

[27] Kreibig U; Vollmer M. Optical Properties of Metal Clusters. Springer Ser.Mater.Sci, vol.25(1995, Springer, Berlin)

[28] Johnson P.B; Christy R.W. Optical constants of the noble metals. Phys.Rev.B,1972,12,4370-4379

[29] Prodan E; Nordander P. Plasmon hybridization in spherical nanoparticles. J.Chem.Phys,2004,120,5444-5454

[30] Deeb C; Zhou X; Grard D; Bouhelier A; Jain P.K; Plain J; Soppera O; Royer P; Bachelot R. Off-resonant optical excitation of gold nanorods:nanoscale imprint of polarization surface charge distribution. J.Phys.Chem.Lett.,2011,2,7-11.

[31] Zhu J; Ren Y.J; Zhao S.M. Refractive index sensitivity of gold double concentric nanoshells: Tuning the intensity discrepancy of two-band plasmonic absorption. Sensors and Actuators B: Chemical,2012,161,1129-1134

[32] Sekhon J.S, Verma S.S. Tunable plasmonic properties of silver nanorods for nanosensing applications. J.Mater.Sci., 2012 , 47,1930-1937 
Figures

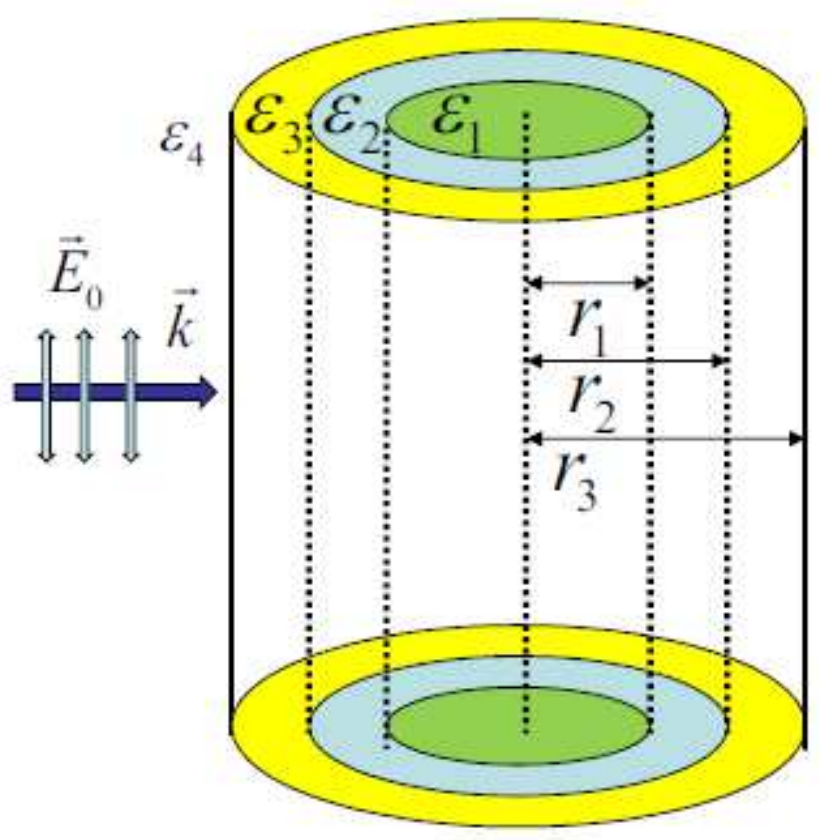

Figure 1

The geometry of gold-dielectric-silver nanotube 

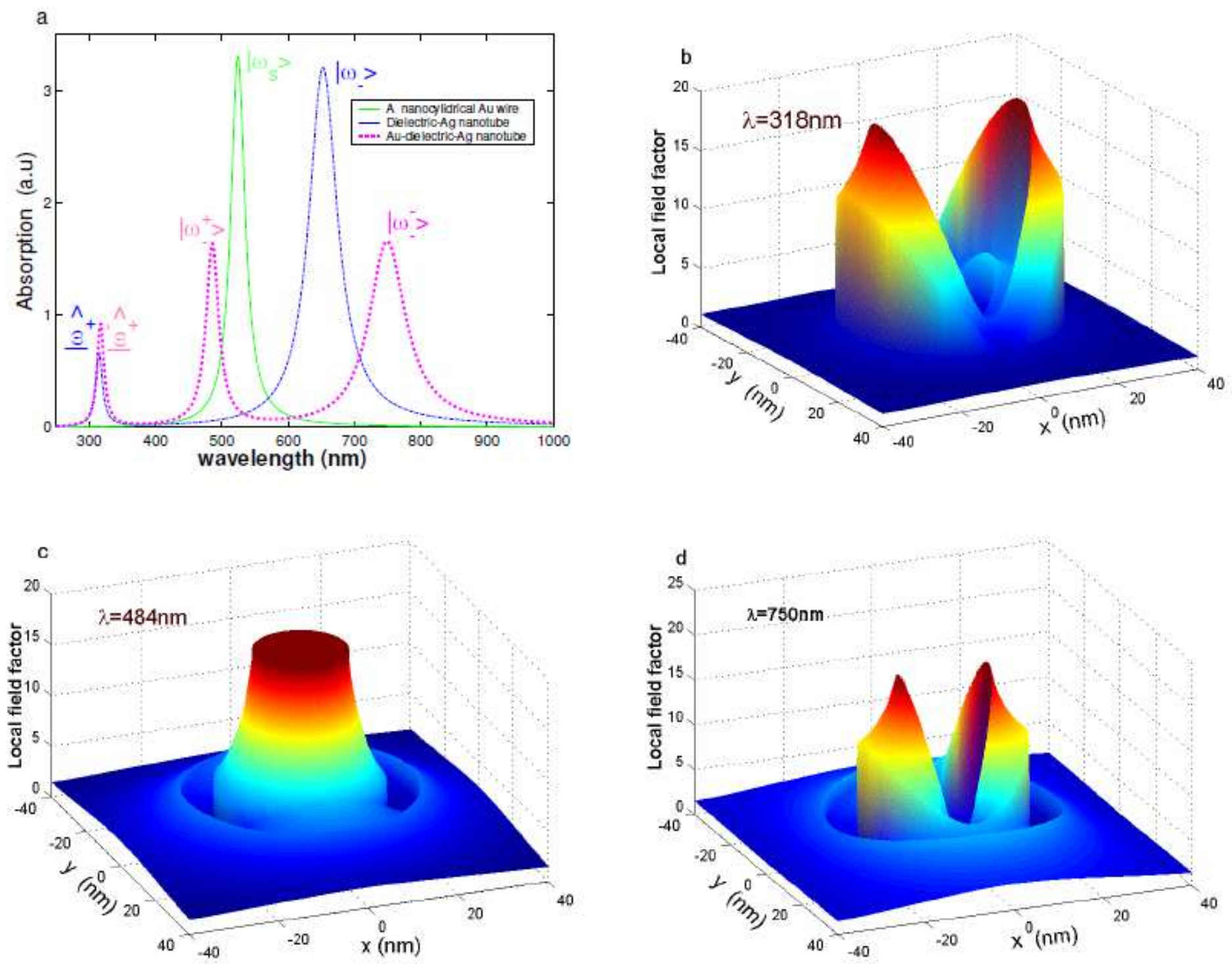

\section{Figure 2}

(a) Absorption spectra of gold-dielectric-silver nanotubes calculated for a single cylindrical gold wire, dielectric-silver nanotube and gold-dielectric-silver nanotubes, $(b, c, d)$ the local electric field intensity distribution of gold-dielectric-silver nanotubes at each LSPR wavelength $\lambda=318 \mathrm{~nm}, \lambda=484 \mathrm{~nm}$ and $\lambda=$ 750nm, respectively. 

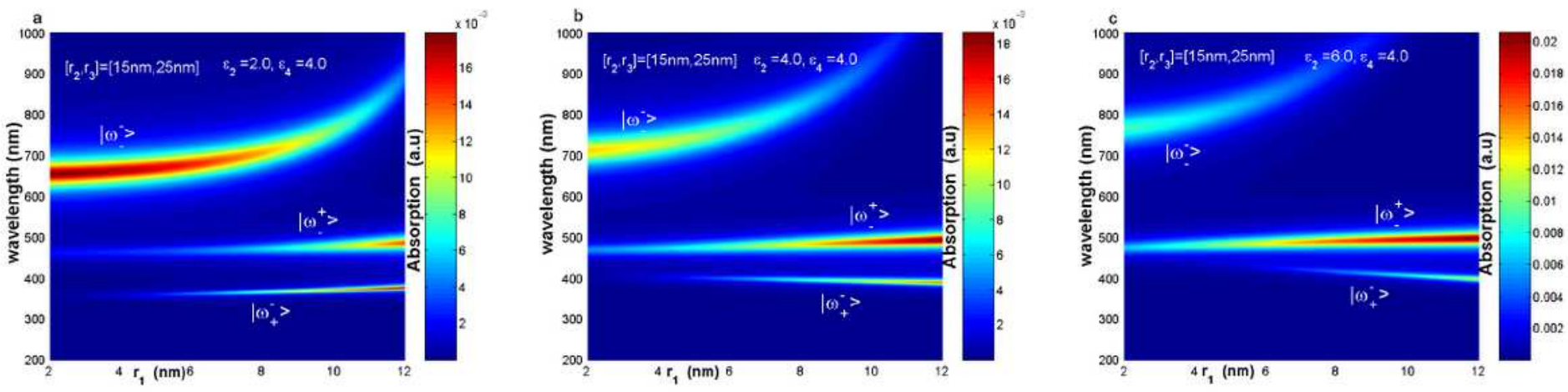

\section{Figure 3}

The effects of inner gold wire radius $\mathrm{r} 1$ on absorption spectra of gold-dielectric-silver nanotube with [r2, $\mathrm{r} 3]=[15 \mathrm{~nm}, 25 \mathrm{~nm}]$ and $\varepsilon 4=4.0$. (a) $\varepsilon 2=2.0$, (b) $\varepsilon 2=4.0$ and (c) $\varepsilon 2=6.0$, respectively.
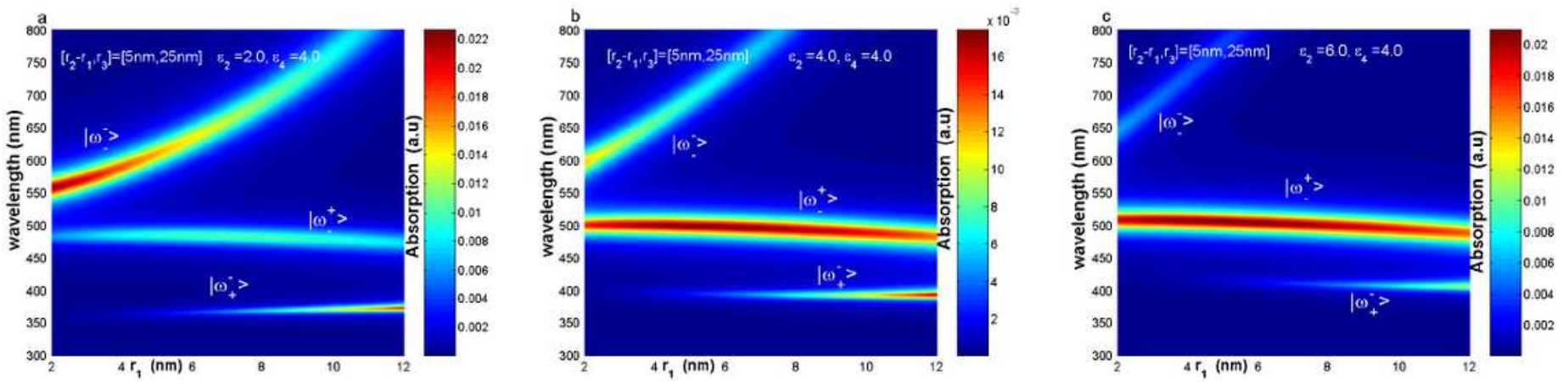

\section{Figure 4}

The effects of inner gold wire radius $r 1$ on absorption spectra of gold-dielectric-silver nanotube with [ $\mathrm{r} 2$ $r 1, r 3]=[5 \mathrm{~nm}, 25 \mathrm{~nm}]$ and $\varepsilon 4=4.0$. (a) $\varepsilon 2=2.0$, (b) $\varepsilon 2=4.0$ and (c) $\varepsilon 2=6.0$, respectively.
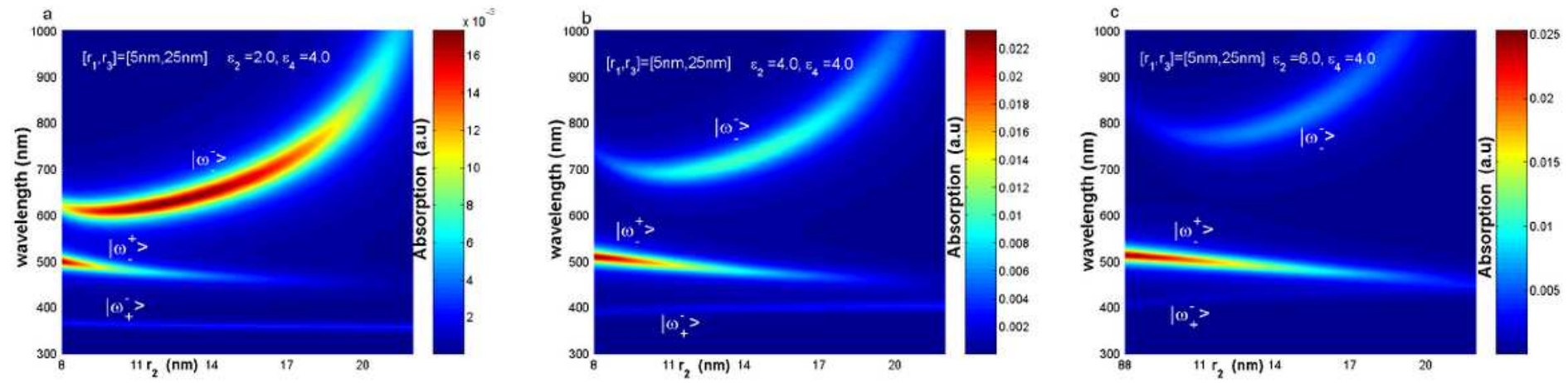

\section{Figure 5}

The effects of middle dielectric radius $\mathrm{r} 2$ on absorption spectra of gold-dielectric-silver nanotube with [ $\mathrm{r}$, $\mathrm{r} 3]=[5 \mathrm{~nm}, 25 \mathrm{~nm}]$ and $\varepsilon 4=4.0$. (a) $\varepsilon 2=2.0$, (b) $\varepsilon 2=4.0$ and (c) $\varepsilon 2=6.0$, respectively. 

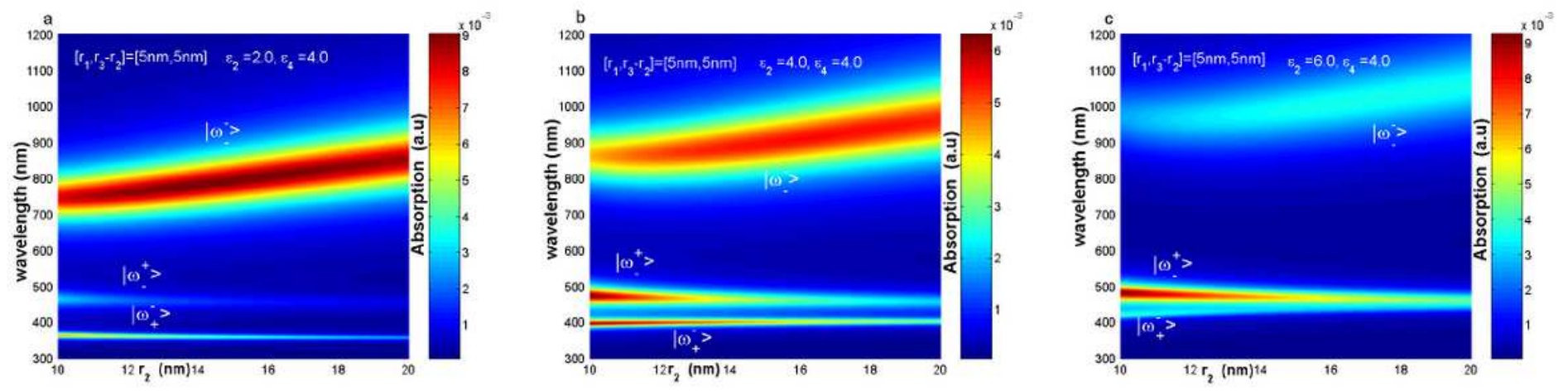

Figure 6

The effects of middle dielectric radius $\mathrm{r} 2$ on absorption spectra of gold-dielectric-silver nanotube with [r1, $\mathrm{r} 3-\mathrm{r} 2]=[5 \mathrm{~nm}, 5 \mathrm{~nm}]$ and $\varepsilon 4=4.0$. (a) $\varepsilon 2=2.0$, (b) $\varepsilon 2=4.0$ and (c) $\varepsilon 2=6.0$, respectively.
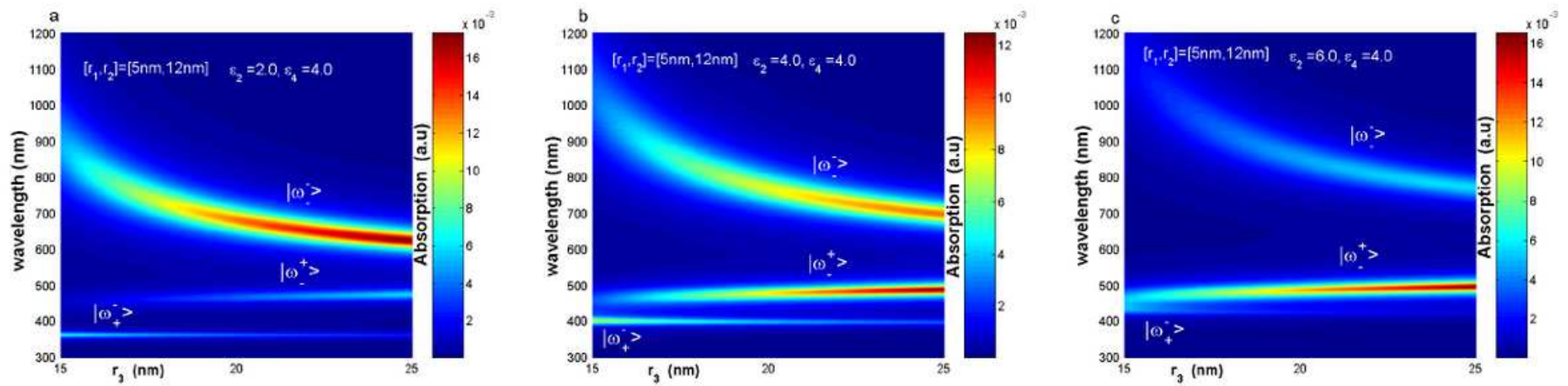

Figure 7

The effects of outer nanotube radius $\mathrm{r} 3$ on absorption spectra of gold-dielectric-silver nanotube with [r1, $\mathrm{r} 2]=[5 \mathrm{~nm}, 10 \mathrm{~nm}]$ and $\varepsilon 4=4.0$. (a) $\varepsilon 2=2.0$, (b) $\varepsilon 2=4.0$ and (c) $\varepsilon 2=6.0$, respectively.
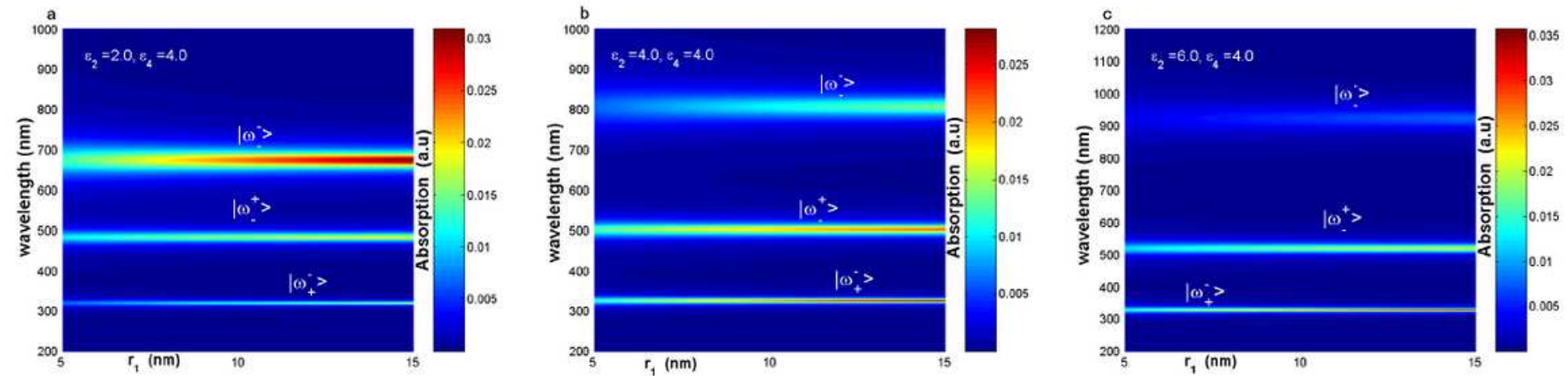

Figure 8 
The effects of the ratio of $\mathrm{r} 1 / \mathrm{r} 2 / \mathrm{r} 3=$ constant on absorption spectra of gold-dielectric-silver nanotube with $\mathrm{r} 1=5 \mathrm{~nm}$ and $\varepsilon 4=4.0$. (a) $\varepsilon 2=2.0$, (b) $\varepsilon 2=4.0$ and (c) $\varepsilon 2=6.0$, respectively.
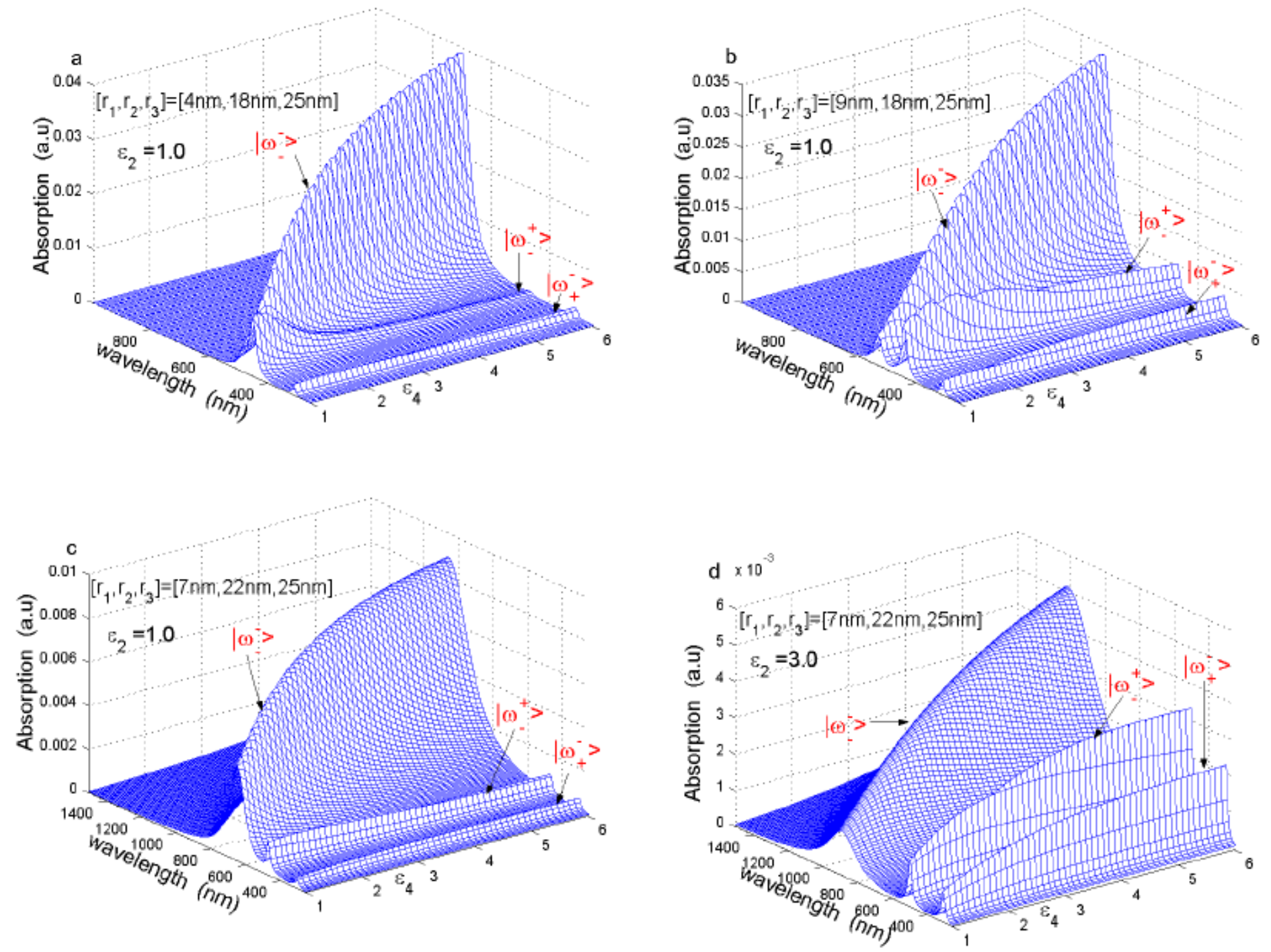

Figure 9

The effects of surrounding medium $\varepsilon 4$ on LSPR of gold-dielectric-silver nanotube. (a) $[\mathrm{r} 1, \mathrm{r} 2, \mathrm{r} 3]=[4 \mathrm{~nm}$, $18 \mathrm{~nm}, 25 \mathrm{~nm}]$ and $\varepsilon 2=1.0$, (b) $[\mathrm{r} 1, \mathrm{r} 2, \mathrm{r} 3]=[9 \mathrm{~nm}, 18 \mathrm{~nm}, 25 \mathrm{~nm}]$ and $\varepsilon 2=1.0,(\mathrm{c})[\mathrm{r} 1, \mathrm{r} 2, \mathrm{r} 3]=[7 \mathrm{~nm}, 22 \mathrm{~nm}$, $25 \mathrm{~nm}]$ and $\varepsilon 2=1.0$, and $(\mathrm{d})[\mathrm{r} 1, \mathrm{r} 2, \mathrm{r} 3]=[7 \mathrm{~nm}, 22 \mathrm{~nm}, 25 \mathrm{~nm}]$ and $\varepsilon 2=3.0$. 

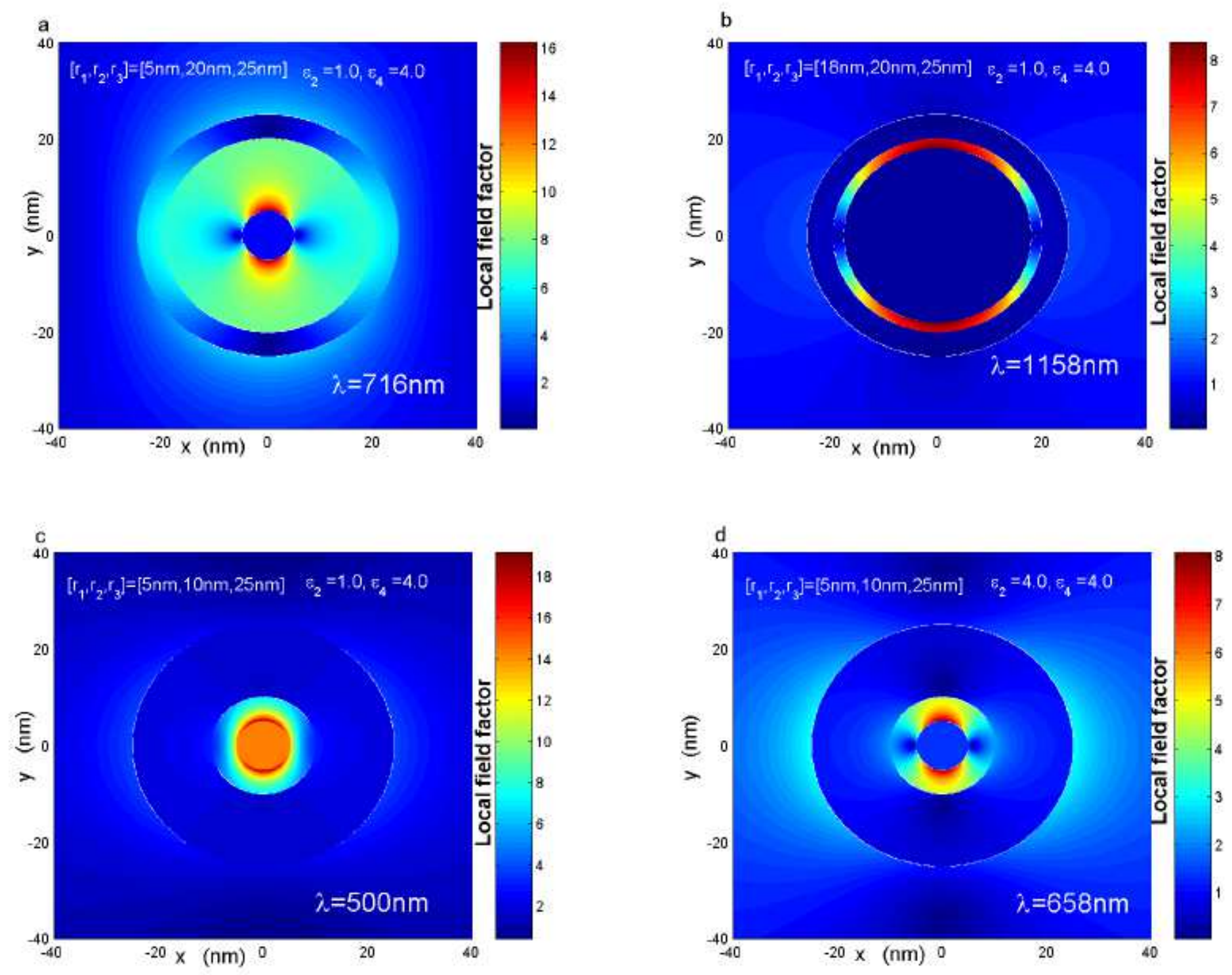

Figure 10

The distributions of local electrical field intensity of gold-dielectric-silver at the resonance wavelength corresponding to $\omega$-> mode with different middle dielectric radii and middle dielectric functions. (a) [r1, r2] $=[5 \mathrm{~nm}, 20 \mathrm{~nm}],[\varepsilon 2, \varepsilon 4]=[1.0,4.0]$ and $\lambda=716 \mathrm{~nm},(b)[\mathrm{r} 1, \mathrm{r} 2]=[18 \mathrm{~nm}, 20 \mathrm{~nm}],[\varepsilon 2, \varepsilon 4]=[1.0,4.0]$ and $\lambda=$ $1158 \mathrm{~nm},(\mathrm{c}[\mathrm{r} 1, \mathrm{r} 2]=[5 \mathrm{~nm}, 2 \mathrm{~nm}],[\varepsilon 2, \varepsilon 4]=[1.0,4.0]$ and $\lambda=500 \mathrm{~nm},(b)[\mathrm{r} 1, \mathrm{r} 2]=[5,10],[\varepsilon 2, \varepsilon 4]=[4.0,4.0]$ and $\lambda=658$ 